\title{
Painless mass in leg: diagnosis and discussion
}

\author{
Francisco Amores-Ramírez • Isabel Hierro Martín • Elvira Montañez Heredia • \\ Pedro Garcia-Fortea • Ana Isabel Garcia Salguero • \\ Antonio Fernandez de Rota Conde
}

Published online: 25 July 2009

(C) ISS 2009

\section{Diagnosis}

Parosteal ossifying lipoma of the fibula: a case of benign lipomatous tumor showing gadolinium enhancement on MRI.

\section{Discussion}

Radiography shows a well-defined, calcified parosteal softtissue mass with fat density and calcification, apparently attached to the fibular cortex. Computed tomography shows both mass components (calcium and fat), well-delimited edges and the cortical relationship with a short narrow pedicle. Magnetic resonance imaging with a gadoliniumenhanced dynamic three-dimensional axial sequence shows inhomogeneity with fat-saturated low signal areas and foci of enhancement in non-fatty areas.

The case presentation can be found at doi:10.1007/s00256-009-0749-z.

We declare that the present research complies with the current laws of the country in which it was done (Spain), and that we have had full control of all primary data.

F. Amores-Ramírez

Department of Radiology, Hospital Virgen de la Victoria, Málaga,

Spain, Campus Universitario de Teatinos s/n,

29010 Málaga, Spain

\section{Hierro Martín}

Department of Pathology, Hospital Virgen de la Victoria,

Campus Universitario de Teatinos $\mathrm{s} / \mathrm{n}$,

29010 Málaga, Spain

\section{E. Montañez Heredia}

Department of Orthopedic Surgery and Traumatology,

Hospital Virgen de la Victoria,

Campus Universitario de Teatinos s/n,

29010 Málaga, Spain
The mass was surgically excised with a wide margin. The gross specimen consisted of an encapsulated, $1.8 \times$ $1.2 \times 1-\mathrm{cm}$ mass with a smooth whitish surface, tightly attached to fibular periosteum. Slicing revealed mainly yellowish, adipose tissue with a few whitish fibrous bands and numerous foci of petrous, osseous tissue. Histologically, the tumor showed mature adipose tissue, embedded strands of fibrous tissue and areas of chondro-osseous tissue with typical endochondral bone formation, forming dispersed foci of mature trabecular bone, with abundant intertrabecular vascularization (Fig. 4 of case presentation). No mitotic figures or areas of hematopoiesis were seen. The final pathologic diagnosis was parosteal ossifying lipoma.

Parosteal ossifying lipoma is a very rare subtype of parosteal lipoma intimately related with the periosteum of the adjacent bone. It contains adipose tissue and extensive areas of cartilaginous and osseous differentiation. Only one case has been reported on the fibula [1]. Four possible radiographic appearances of parosteal lipoma have been described, depending on the association with chondroid and/or osseous modulation [2]. The lipoma may rest

P. Garcia-Fortea $(\bowtie)$

Subdirector Médico, Hospital Materno-Infantil (HRU Carlos Haya), Calle Isaías Carrasco Miguel 24,

29620 Málaga, Spain

e-mail: pgfortea@yahoo.com

A. I. Garcia Salguero

Department of Pathology, Hospital Virgen de la Victoria,

Campus Universitario de Teatinos $\mathrm{s} / \mathrm{n}$,

29010 Málaga, Spain

A. Fernandez de Rota Conde

Department of Orthopedic Surgery and Traumatology,

Hospital Virgen de la Victoria, Campus Universitario de Teatinos s/n, 29010 Málaga, Spain 
directly on the cortex with no elements of cartilage or bone, or with patches of chondroid and bone tissue scattered throughout the lipomatous mass. It may have a narrow bony stalk with a lucent lipomatous cap mimicking a pedunculated exostosis, or a densely ossified broad-based osteochondromatous element beneath a thin lipomatous cap simulating a sessile exostosis. The differential diagnosis includes other benign fatty tumors, myositis ossificans, florid hyperostosis, synovial sarcoma and low grade liposarcoma.

Panzarella et al. [3] determined the predictive value of preoperative gadolinium-enhanced MRI in distinguishing 32 well-differentiated liposarcomas from benign fatty tumors. As a predictor of liposarcoma, the presence of gadolinium enhancement showed $100 \%$ sensitivity, $71 \%$ specificity, $53 \%$ positive predictive value and $100 \%$ negative predictive value. Gadolinium enhancement of thick septa and/or nodular or globular foci in a mostly homogeneous, fatty, soft tissue tumor is a sensitive screening method to determine the diagnosis of a welldifferentiated liposarcoma. However, as a predictor of liposarcoma, false positive findings affect its specificity and positive predictive value. This occurs with gadoliniumenhanced MRI of benign fatty tumors (angiolipoma, fibrolipoma, hibernoma and ossifying chondroid lipoma), due to their increased vascularity [3-5]. This first report of a gadolinium-enhanced MRI of parosteal ossifying lipoma shows that it should also be considered in this group of benign lipomatous tumors. Nevertheless, the typical radiographic appearance, CT and MRI features should be sufficient to diagnose a parosteal ossifying lipoma with no further contrast studies, as signal enhancement does not contradict its benign nature nor modify its differential diagnosis or therapeutic approach.

\section{References}

1. Bui-Mansfield LT, Myers CP, Chew FS. Parosteal lipoma of the fibula. AJR Am J Roentgenol. 2000;174(6):1698.

2. Miller MD, Ragsdale BD, Sweet DE. Parosteal lipomas: a new perspective. Pathology (Phila). 1992;24(3):132-9.

3. Panzarella MJ, Naqvi AH, Cohen HE, Damron TA. Predictive value of gadolinium enhancement in differentiating ALT/WD liposarcomas from benign fatty tumors. Skeletal Radiol. 2005;34 (5):272-8.

4. Val-Bernal JF, Val D, Garijo MF, Vega A, Gonzalez-Vela MC. Subcutaneous ossifying lipoma: case report and review of the literature. J Cutan Pathol. 2007;34(10):788-92.

5. Hoch B, Hermann G, Klein MJ, Abdelwahab IF. Ossifying chondroid lipoma. Skeletal Radiol. 2008;37(5):475-80. 